ARTICLE

\title{
Local pH oscillations witness autocatalytic self-organization of biomorphic nanostructures
}

\author{
M. Montalti', G. Zhang'², D. Genovese' ${ }^{1}$ J. Morales², M. Kellermeier ${ }^{3} \&$ J.M. García-Ruiz ${ }^{2}$
}

Bottom-up self-assembly of simple molecular compounds is a prime pathway to complex materials with interesting structures and functions. Coupled reaction systems are known to spontaneously produce highly ordered patterns, so far observed in soft matter. Here we show that similar phenomena can occur during silica-carbonate crystallization, the emerging order being preserved. The resulting materials, called silica biomorphs, exhibit non-crystallographic curved morphologies and hierarchical textures, much reminiscent of structural principles found in natural biominerals. We have used a fluorescent chemosensor to probe local conditions during the growth of such self-organized nanostructures. We demonstrate that the $\mathrm{pH}$ oscillates in the local microenvironment near the growth front due to chemical coupling, which becomes manifest in the final mineralized architectures as intrinsic banding patterns with the same periodicity. A better understanding of dynamic autocatalytic crystallization processes in such simple model systems is key to the rational development of advanced materials and to unravel the mechanisms of biomineralization.

\footnotetext{
${ }^{1}$ Dipartimento di Chimica 'G. Ciamician', Alma Mater Studiorum, Università di Bologna, I-40126 Bologna, Italy. ${ }^{2}$ Laboratorio de Estudios Cristalográficos, Instituto Andaluz de Ciencias de la Tierra (CSIC-Universidad de Granada), E-18100 Armilla (Granada), Spain. ${ }^{3}$ Material Physics, BASF SE, RAA/OS-B007, D-67056 Ludwigshafen, Germany. Correspondence and requests for materials should be addressed to J.M.G.-R. (email: juanmanuel.garcia@csic.es).
} 
iving organisms are able to precisely control the formation of crystalline matter throughout their tissues, typically by using organic (macro)molecules that direct the assembly of the inorganic component into architectures with exceptionally complex shapes and textures ${ }^{1-3}$. Therefore, it is not surprising that most attempts to mimic biomineralization in vitro have relied on organic molecules ${ }^{4}$ and investigated their effect on the nucleation and growth of prominent minerals like calcium carbonate $^{5}$ or phosphate ${ }^{6}$. By contrast, the inorganic-inorganic route for the formation of hierarchical structures has scarcely been explored. Yet, bizarre as it might appear, it has been shown that materials with features closely resembling those of biominerals can be obtained in simple single-pot experiments ${ }^{7}$. These so-called silica biomorphs form in alkaline media upon crystallization of carbonates like $\mathrm{BaCO}_{3}$ or $\mathrm{CaCO}_{3}$ under the influence of dissolved silicate species ${ }^{8-10}$. This process results in completely inorganic nanostructures that display (i) complex non-crystallographic morphologies with continuous curvature (such as regular helicoids, Fig. 1a) (refs 8-10); (ii) mesocrystaltype textures with long-range orientational order (Fig. 1b) (refs 9,11,12); (iii) banded patterns (Fig. 1c,d) (refs 7,13,14) and
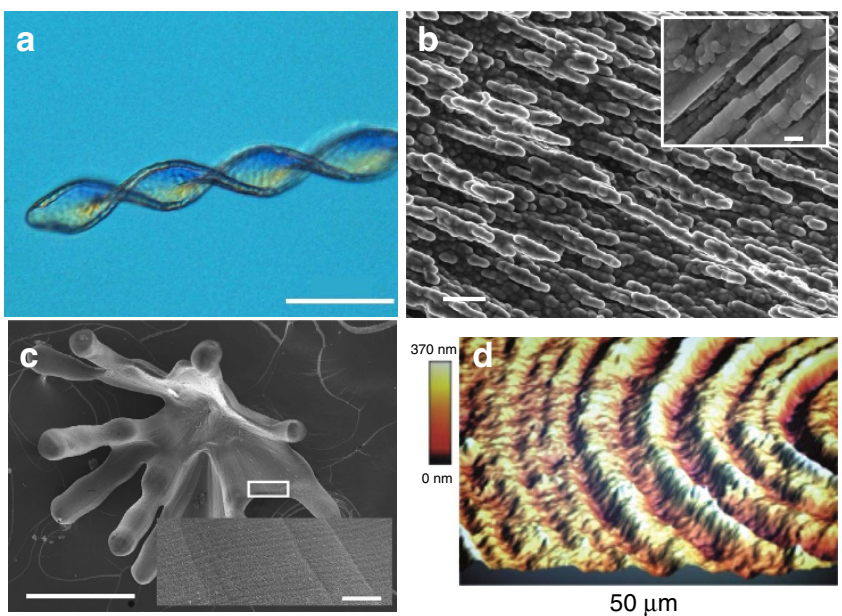

$50 \mu \mathrm{m}$
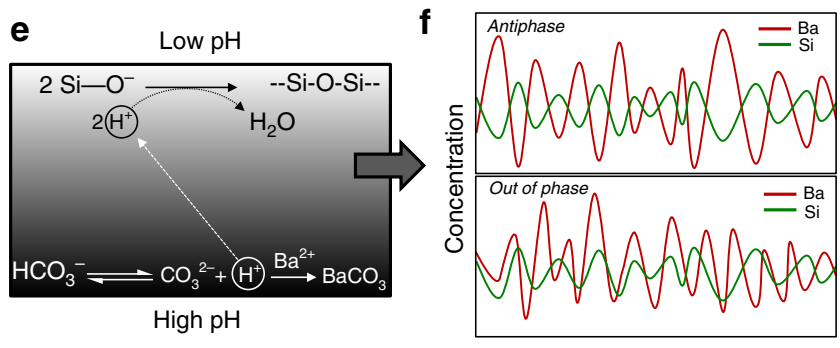

Distance

\section{Figure 1 | Complex self-assembly in purely inorganic systems.}

(a) Polarized optical micrograph of a barium carbonate biomorph with twisted morphology. (b) High-magnification SEM image revealing that biomorphs consist of numerous co-oriented carbonate nanocrystals (in this case aragonite). These nanorods grow by accretion of still smaller spherical subunits, as shown in the inset. (c) SEM image of a calcium carbonate biomorph (here monohydrocalcite) exhibiting periodic banding patterns (as seen in the magnified inset). (d) 3D reconstruction of an AFM height profile of a biomorph with sheet-like structure where periodic banding is clearly evident. (e) Scheme of the coupled chemical reactions leading to alternating precipitation of barium carbonate and silica during the formation of biomorphs. (f) Sketch of two different types of spatial composition banding that could arise from the model depicted in e. Scale bars: (a) $50 \mu \mathrm{m}$; (b) $1 \mu \mathrm{m}$; inset $100 \mathrm{~nm}$; (c) $300 \mu \mathrm{m}$; inset, $10 \mu \mathrm{m}$. (iv) structural features indicative of a growth mechanism involving the accretion of amorphous primary particles (inset in Fig. 1b) (ref. 15).

Given these striking analogies to natural biominerals and their relevance for material science ${ }^{16}$ and early life detection ${ }^{8,17}$, considerable effort has been made to understand the formation of silica biomorphs ${ }^{7-10,14,18-21}$. The current picture assumes a coupling of the chemical speciation of silicic and carbonic acid in solution, which drives the concerted precipitation of the two mineral components across all relevant length scales ${ }^{9-10,19-21}$. The model (schematically depicted in Fig. 1e,f) is based on the inverse $\mathrm{pH}$ dependence of the solubility of carbonate and silica: as carbonate crystals form at the front of a growing biomorph, the accompanying release of protons provokes a local decrease of $\mathrm{pH}$ and induces the precipitation of silica, which inhibits further carbonate growth. In turn, silica polycondensation causes an increase in $\mathrm{pH}$ and therefore triggers a new event of carbonate nucleation. In this way, the system maintains an autocatalytic cycle of co-precipitation, during which silica-coated carbonate nanocrystals are produced continuously and self-organize into two-dimensional surfaces that may later bend and twist to develop delicate helical or worm-like morphologies $9-10,20-21$.

An immediate consequence of the envisaged growth mechanism is that the local $\mathrm{pH}$ near the active front should oscillate in time and space. Although there is evidence supporting the notion of local speciation changes ${ }^{19,22}$, no direct proof for the oscillatory behaviour-and hence the autocatalytic nature of the processhas been achieved so far. The main difficulty for the detection of the predicted local variations in $\mathrm{pH}$ is the short length and time scales at which changes are expected to occur, and that absolute differences in $\mathrm{pH}$ are likely to be very small. In the present work, we have solved this problem by using fluorescence microscopy, a non-invasive in situ technique with high sensitivity ${ }^{23,24}$. The mapping of $\mathrm{pH}$ by fluorescence imaging requires the presence of a suitable $\mathrm{pH}$-sensitive fluorescent chemosensor in the reaction medium. Here we have used acridine orange (Fig. 2a), a dye that gives almost no fluorescence signal in its neutral form (AO) prevailing at the typical bulk $\mathrm{pH}(\sim 11.0)$ in our experiments, whereas a local decrease in $\mathrm{pH}$ will increase the concentration of the strongly fluorescent cationic form (AOH) (ref. 25). Consequently, the fluorescence signal at a given position provides direct information about the $\mathrm{pH}$ in the local microenvironment.

\section{Results}

Mapping $\mathbf{p H}$ by fluorescence microscopy. Figure $2 \mathrm{~b}$ shows a sequence of fluorescence images collected from a biomorph aggregate that grew flat (two-dimensional sheet) and perpendicular to the optical axis of the microscope (see in the Fluorescence Microscopy section in the Supplementary Information for details on experimental methods and Movie 1 for the whole recorded time-lapse sequence of the growing structure). The bright fluorescence observed at the rim of the aggregate reveals enhanced protonation of the chemosensor and shows that the $\mathrm{pH}$ is indeed decreased in the proximity of the growth front relative to the surrounding alkaline bulk. In order to analyse the propagation of the fluorescence intensity in space, the time-lapse images were overlapped (Fig. 2c) and eight representative directions (D1-D8) along the growth vectors were selected to track the position of the fluorescence as well as its intensity. Figure $2 \mathrm{~d}$ shows the intensity profiles along the growth vector D1 at different times, from which three important conclusions can be drawn. First, the position of the fluorescence peak moves linearly with time (Fig. $2 \mathrm{~g}$ ) at a rate of $0.21 \mu \mathrm{m} \mathrm{min}^{-1}$, which is in a range typical for the growth of biomorphs ${ }^{9,20}$ and thus confirms that the fluorescence peak 
a<smiles>C[P+]#CC#CN(C)c1ccc2cc3ccc(N(C)C)cc3nc2c1</smiles>

b

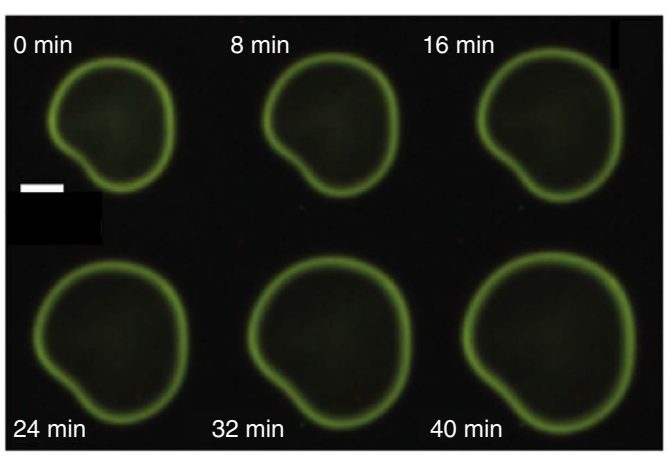

c

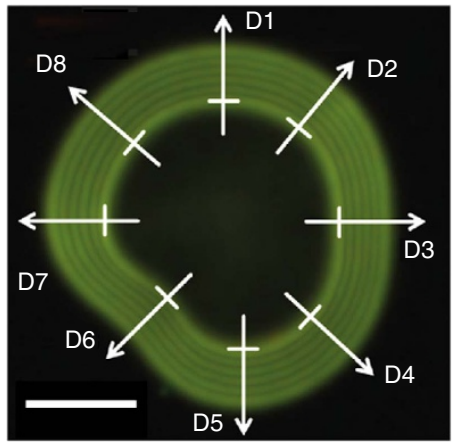

d
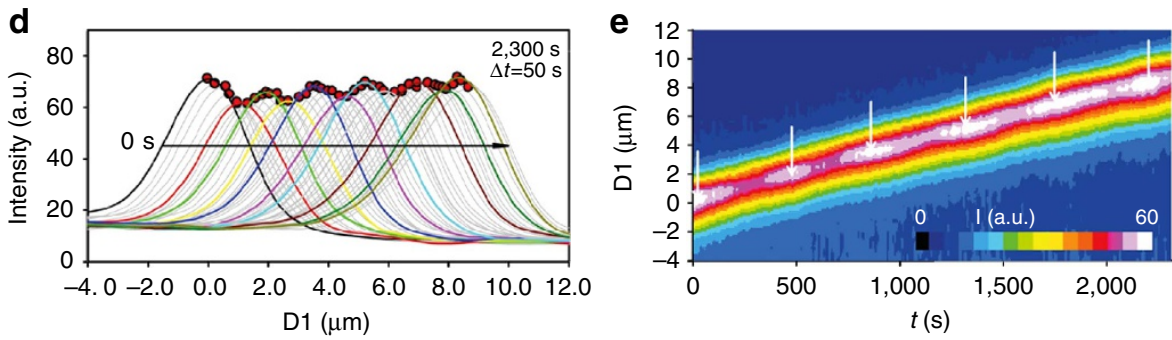

f
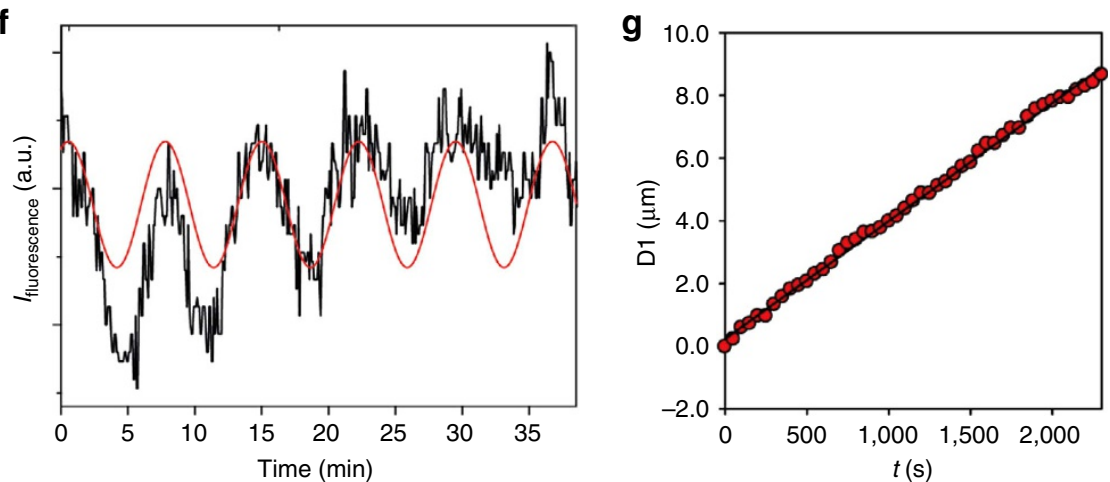

Figure 2 | In situ measurements of local pH variations during the formation of silica biomorphs. (a) Molecular structure of acridine orange in its neutral $(\mathrm{AO})$ and protonated state $\left(\mathrm{AOH}^{+}\right)$. (b) Fluorescence images acquired at different times (as indicated) from a biomorph sheet growing in the presence of acridine orange $\left(50 \mu \mathrm{M}, \lambda_{\text {exc. }}=488 \mathrm{~nm}\right)$. (c) Overlap of the shown images in c with eight selected directions along the respective growth vectors.

(d) Fluorescence intensity profiles along the growth vector D1 at different times ( $50 \mathrm{~s}$ interval). Curves corresponding to relative intensity maxima and minima are plotted as thicker coloured lines. (e) Contour map showing the evolution of the fluorescence intensity along vector D1 with time. Arrows point to the periodically observed maxima. (f) Plot of the absolute intensity at the fluorescence peak maxima in $\mathbf{d}$ as a function of time. The red line is a sine function fitted to the experimental data. ( $(\mathbf{g})$ Time-dependent change of the position of the fluorescence peak maxima in $\mathbf{d}$. The black line is a linear fit of the data, from which the rate of advance of the reaction front can be obtained. Both the trend and the absolute rate values agree well with data reported for silica biomorphs in the literature 9,20 . Scale bars: $10 \mu \mathrm{m}$.

advances along with the growth front. Second, the peak shape and width do not change significantly with time, demonstrating that the $\mathrm{pH}$ variation occurs within a confined region extending ca. $2 \mu \mathrm{m}$ beyond the growing front, in good agreement with indirect estimations ${ }^{19}$ and recent experimental measurements ${ }^{22}$. The third and most important observation is that the absolute value of the fluorescence intensity at the peak maximum is not constant over time, but clearly shows oscillatory behaviour. This becomes evident when the intensity profiles of all 467 collected frames are combined together in the form of a contour map (Fig. 2e), or when plotting the absolute intensity at the fluorescence peak maximum as a function of time (Fig. 2f). During the monitored period (2,200 s), five complete oscillations (six maxima) can be observed over a total distance of $8.1 \mu \mathrm{m}$, giving an average peak-to-peak separation of $1.5 \mu \mathrm{m}$ and an oscillation frequency of $0.14 \mathrm{~min}^{-1}$ (see the SI for corresponding analyses along the vectors D2-D8).

These results provide clear evidence for the existence of local $\mathrm{pH}$ oscillations at the growth front of silica biomorphs. Calibrations of the measured fluorescence intensities (see Fluorescence microscopy section in the Supplementary Information) suggest that the average $\mathrm{pH}$ in the local microenvironment is about 9.7 (that is, significantly lower than in the bulk, as expected), while the amplitude of the $\mathrm{pH}$ oscillation was 

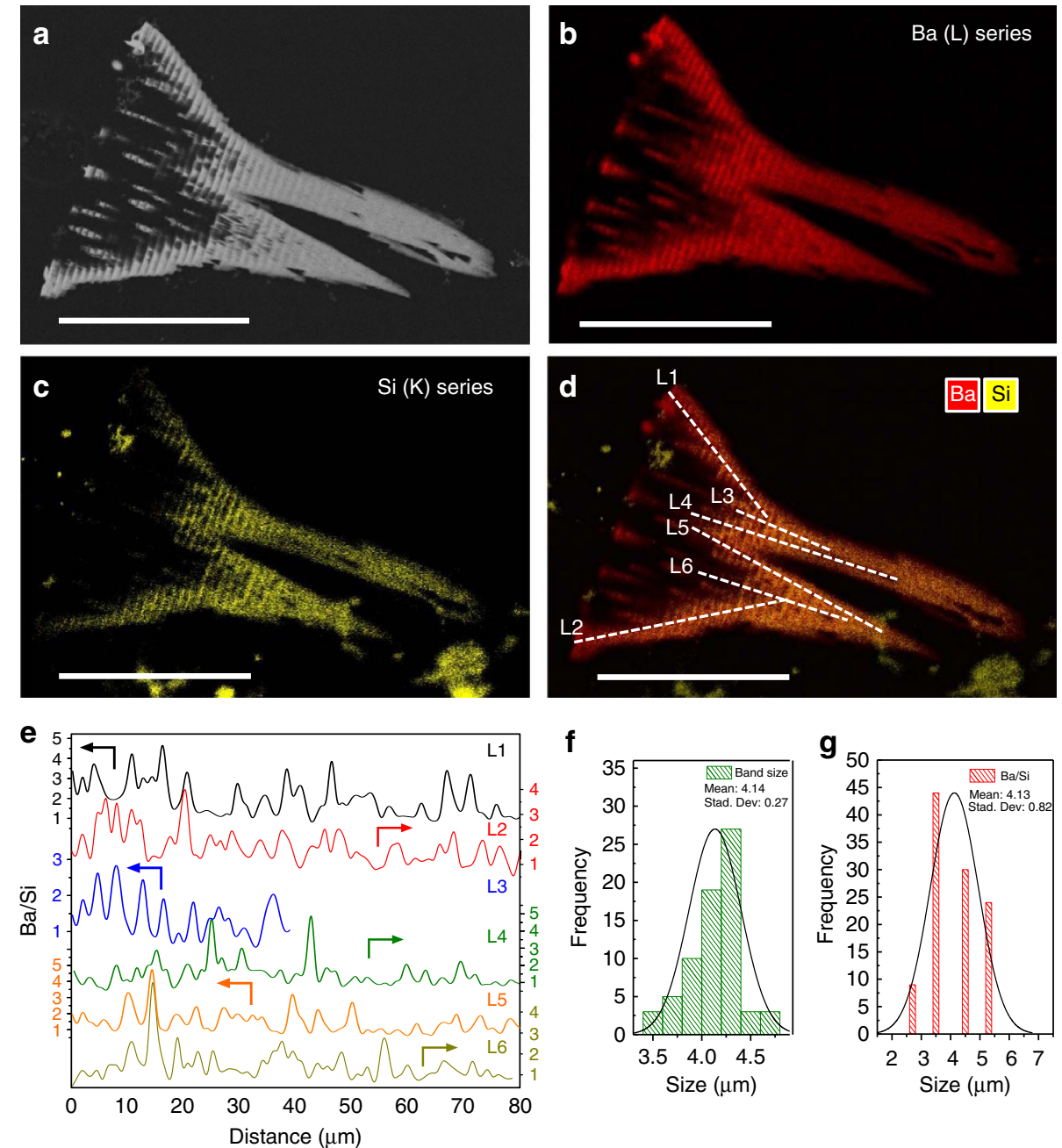

Figure 3 | Chemical composition oscillations in silica biomorphs. (a) Backscattered electron image taken from a thin section of a biomorph with trumpet-like morphology. (b) EDX mapping of the Ba distribution. (c) EDX mapping of the Si distribution. (d) Superposition of the Ba (red) and Si (yellow) signals. White lines represent the paths along which the composition was analysed. (e) Variation of the Ba/Si ratio along the different lines in d. (f) Histogram of the width of the structural banding observed in $\mathbf{a}$. (g) Histogram of the width of the compositional banding observed in e. Scale bars are $100 \mu \mathrm{m}$.

estimated to be ca. 0.2 units relative to the average value. In terms of supersaturation, this difference of 0.2 units of $\mathrm{pH}$ means a saturation index of 1.26 for silica and 1.44 for carbonate, assuming that system is closed. If open to the air, that value increases to 2.5. These values are large enough to trigger nucleation and growth of slightly soluble compounds such as $\mathrm{BaCO}_{3}$ and $\mathrm{SiO}_{2}$. The precise periodicity and scale of the oscillation wavelength exclude any external perturbation as possible reason for the observed trends, and rather imply that the detected periodic variations are intrinsic to the growth process, as also suggested by recent experiments in single-phase systems ${ }^{26}$.

Analysis of self-assembled banding structures. In principle, the observed oscillatory behaviour could be the result of either classical reaction-diffusion coupling (like for example in Liesegang rings $)^{7,27}$, or reaction-reaction coupling as shown in Fig. 1e. While rhythmic banding patterns would be expected in both cases, the latter scenario should in addition lead to periodic changes in composition, as illustrated in Fig. 1f. In order to address this issue, we have performed ex-situ analyses on biomorphs grown with the same protocol as in the fluorescence microscopy experiments. Atomic force microscopy (AFM) images of sheet-like aggregates reveal a wavy topography with periodic variations in thickness on a length scale ranging from 1 to $6 \mu \mathrm{m}$ (Fig. 1d), in good agreement with previous studies on biomorphs of barium carbonate ${ }^{14,26}$ and monohydrocalcite ${ }^{28}$. In all these cases, the periodicity of the banding patterns matches well the frequency of the $\mathrm{pH}$ oscillation observed by fluorescence microscopy, and moreover the banding does not show the squareroot dependence on time typical for reaction-diffusion systems ${ }^{27}$. Most probably, thin regions are formed in phases where the local $\mathrm{pH}$ is low (depressing carbonate crystallization and enhancing silica precipitation), while thicker parts correspond to higher $\mathrm{pH}$ values in the microenvironment.

So far, spatial oscillations in chemical composition as expected for this scenario have not been observed for intact biomorphs ${ }^{14}$. Here, we have probed the composition after polishing the structures, in order to get access to their internal volume and thus to avoid interferences of the outer silica skin that usually covers the biomorphs ${ }^{20}$. Elemental mapping of the inner part of the aggregates shows a clear picture (Fig. 3): the backscattered electron image (Fig. 3a) as well as the signals for both Ba (Fig. 3b) and $\mathrm{Si}$ (Fig. 3c) display periodic variations along the respective growth direction. More importantly, this periodicity is still 
evident in profiles of the $\mathrm{Ba} / \mathrm{Si}$ atomic ratio along different chosen growth vectors (Fig. 3d,e), demonstrating that the local composition in fact oscillates around some average value. In most regions, the concentration of barium is significantly higher than that of silica (usually by a factor of up to 5 ), indicating that $\mathrm{BaCO}_{3}$ precipitation dominates the process, in line with previous observations $^{14,20,22}$. Statistical data analysis confirms that the mean width of the observed bands is about $4 \mu \mathrm{m}$ (Fig. 3f) and that the oscillation in composition has the same frequency (Fig. 3g), both again well in line with the local variations in $\mathrm{pH}$ traced in situ during growth (Fig. 2f). When analysing these composition profiles in more detail, it appears that the oscillations in silica and barium content are not exactly in antiphase (as anticipated in Fig. 1f), but clearly they are out of phase. This shows that the mineralization of the two components is not independent, likely because carbonate and silicate influence each other through their solution speciation as depicted in Fig. 1e.

\section{Discussion}

Our results show that the coupling of carbonate and silicate precipitation induces periodic behaviour at the growth front, which leads to oscillations in local conditions that are directly correlated with topographic and compositional banding patterns found in the final mineralized structures. Autocatalytic processes like those described here are well established in the field of solution chemistry, where the coupling of simple reactions can lead to temporary out-of-equilibrium patterns as in the case of the famous Belousov-Zhabotinsky systems ${ }^{29}$. It has also been reported that the order created in this way can be preserved in the form of $2 \mathrm{D}$ concentric ring structures when one of the involved components mineralizes in the course of the process ${ }^{30}$. The present study shows that reaction coupling of two co-precipitating phases is able to drive the self-organization of much more complex 3D architectures that display structural features otherwise only known from biologically controlled crystallization. Subtle changes in local conditions have proven to be key to the bottom-up assembly of elaborate hierarchical textures, and it may well be envisaged that related effects play a much greater role in natural biomineralization than hitherto believed.

\section{Methods}

Growth of silica biomorphs. The synthesis of silica-barium carbonate biomorphs was performed in aqueous solution following previously established protocols $^{5,20,21}$. First, $1 \mathrm{ml}$ of commercial water glass (Sigma-Aldrich, reagent grade, Ref. 338443 ) was diluted in $350 \mathrm{ml}$ water. The resulting solution was mixed with a small amount of $0.1 \mathrm{M} \mathrm{NaOH}$ to set the $\mathrm{pH}$ to approximately 11 . Then an equal volume of $0.01 \mathrm{M} \mathrm{BaCl}_{2}$ solution was mixed in the diluted water glass solution, which results in a final mixture with a $\mathrm{pH}$ value of $10.6 \pm 0.2$ that contains $5 \mathrm{mM} \mathrm{Ba}$ and $7.5 \mathrm{mM} \mathrm{SiO}_{2}$. Finally, $10 \mathrm{ml}$ of this mixture were combined with $100 \mu \mathrm{l}$ of a $1 \mathrm{mM}$ solution of acridine orange (Sigma-Aldrich) in water.

Crystallization of barium carbonate was triggered by the diffusion of atmospheric carbon dioxide into the above-described alkaline solution ${ }^{21}$. In order to minimize background fluorescence and thus maximize spatial resolution, the experiments were performed in thin crystallization cells with a thickness of

$\sim 100 \mu \mathrm{m}$ as shown in Supplementary Fig. 1: a thin layer of growth solution was sandwiched between a glass slide and a coverslip that were separated by a spacer with two open holes for the uptake of $\mathrm{CO}_{2}$. In particular, two strips of double-sided Scotch tape $(5 \times 40 \mathrm{~mm})$ were attached parallel and close to the two longer edges of a microscopy slide $(75 \times 25 \mathrm{~mm})$ at a distance of $15 \mathrm{~mm}$ from each other. Then $100 \mu \mathrm{l}$ of growth solution was dropped into the centre and a glass coverslip $(40 \times 25 \mathrm{~mm})$ was placed on the free side of the tape to close the cell. This configuration allowed for an optimized spatial resolution in the fluorescence signal, as the thickness of the layer of bulk solution illuminated during the experiment and hence the background emission was reduced (without any noticeable interference with the growth of biomorphs). In addition, the use of thin growth cells minimizes convective flow so that the biomorphs grow with little perturbation at a given location, facilitating measurements of their growth rate.
Fluorescence microscopy. UV-Vis absorption and fluorescence measurements were performed using a PerkinElmer Lambda $25 \mathrm{UV}-\mathrm{V}$ is spectrophotometer and a FluoroMax-4 (HORIBA) spectrofluorimeter respectively. The growth of the biomorphs was monitored in situ by wide-field fluorescence microscopy (Olympus IX 71 inverted microscope) under low-power excitation conditions. The wide-field illumination for excitation was achieved by focusing the expanded and collimated laser beam (Argon laser, Melles-Griot 534 Series, $\lambda_{\text {exc }}=488 \mathrm{~nm}$ ) onto the backfocal plane of the objective (UPLFLN10X2, Köhler illumination mode) using a suitable dichroic mirror (Chrome 500dcxr). In this experimental setup, the dichroic mirror irradiated the specimen with the desired wavelength and then separated the much weaker emitted light (that is, the fluorescence) from the excitation light. After being further filtered, the emitted light reached the eye or the detector so that the fluorescent areas were contrasted against a dark background. The detection limit is largely determined by the darkness of the background, of which the emitted light should typically be $10^{5}$ or $10^{6}$ times weaker than the excitation light. Fluorescent images were collected using a CCD camera (Basler Scout scA 640-70) and further converted into videos using ImageJ to visualize the growth of biomorphs with fluorescence contrast. Videos were recorded during the first $12 \mathrm{~h}$ of the experiments, with a typical interval between frames of $5 \mathrm{~s}$.

In order to convert the measured intensity data into actual (local) $\mathrm{pH}$ values, a calibration curve was acquired using $10^{-5} \mathrm{M}$ solutions of $\mathrm{AO}$ in water, which were adjusted to different $\mathrm{pH}$ levels by adding small volumes of either $\mathrm{HCl}$ or $\mathrm{NaOH}$. Fluorescence intensity was measured with the same spectrofluorimeter as used for the growth experiments, while $\mathrm{pH}$ was monitored in parallel with a Jenway $4330 \mathrm{pH}$-meter equipped with a Crison sensor. The resulting dependence of the fluorescence intensity $I$ on $\mathrm{pH}$ is shown in Supplementary Fig. 3. The experimental data were fitted with the following equation:

$$
I=I_{2}+\frac{I_{1}-I_{2}}{1+10^{-\left(p k_{a}-p H\right)}}
$$

where $I_{1}$ and $I_{2}$ are the intensities measured for AO solutions at $\mathrm{pH} 7.0$ and 13.0, respectively. This gave a pKa value of $10.0 \pm 0.1$ for the AO dye. Using this calibration curve, the fluorescence intensities measured in the growth experiments were converted into $\mathrm{pH}$ values according to:

$$
p H=p k_{a}+\log \left(\frac{I_{1}-I}{I-I_{2}}\right)
$$

The spacial resolution of the fluorescence intensity measurements by microscopy was $0.9 \mu \mathrm{m}$ for the experimental set up. The fluorescence images shown in Fig. $2 \mathrm{~b}$ reveal that a small fraction of the $\mathrm{AO}$ dye is incorporated in the biomorph during growth. Considering the fluorescence intensity in the inner part of the crystal aggregates, where growth is terminated, we could estimate the concentration of the dye in the biomorph to be comparable with that in the silicate solution (i.e. $10^{-5} \mathrm{M}$ ) and hence the content of AO in the biomorphs was less than 3 p.p.m.. Such low concentrations can hardly affect precipitation, and indeed we observed very similar growth rates values, growth rate trend, shapes and sizes of biomorphs with and without the dye. For instance, the growth of biomorphs in our fluorescence experiments was found to be linear in time and the growth rate to vary in the order of tens of microns per hour (Fig. 2e), in good agreement with previous experiments. In addition, the wavelength of the $\mathrm{pH}$ oscillations measured in our fluorescence experiments ( 1.5 microns) is similar to the wavelength of textural and compositional banding ( 4.1 microns) in the final structures obtained in this work (Fig. 3f,g) as well as in previous studies on $\mathrm{MHC}$ biomorphs ${ }^{28}$ and $\mathrm{BaCO}_{3}$ biomorphs ${ }^{15,26}$ (that is, in the range between 1 and 6 microns).

Ex-situ characterization. Biomorphs grown according to the above-described protocol (with or without the fluorescent dye) were isolated by removing the mother solution after predefined times, followed by repeated rinsing with water and ultimately ethanol. Optical micrographs of the resulting structures were taken using a Nikon AZ100 microscope. For AFM, small glass slides were added to the growth solution as substrates. Biomorphs formed thereon remained stuck even upon extensive rinsing and thus they could be directly investigated after drying. AFM studies were performed on an Autoprobe CP microscope (Park Scientific Instruments) in non-contact mode using cantilevers (non-contact ultralevers, ULCN-AUMT-A) with a resonance frequency of $80 \mathrm{kHz}$. Finally, for scanning electron microscopy (SEM) and elemental analyses by energy-dispersive X-ray (EDX) spectroscopy, suitable biomorphic structures were selected under a stereomicroscope and embedded within epoxy resin using a glass slide as substrate. The resulting specimen was sequentially polished with diamond powder up to a grain size of $1 \mu \mathrm{m}$ until the internal structure of the biomorphs was exposed at the surface. The sample was eventually coated with carbon and studied by SEM using a Zeiss SUPRA $40 \mathrm{VP}$ microscope equipped with an X-Max $50 \mathrm{~mm}$ EDX system from Oxford Instruments.

Data availability. The authors declare that the data supporting the findings of this study are available within the paper and its Supplementary Information files. 


\section{References}

1. Weiner, S. \& Addadi, L. Crystallization pathways in biomineralization. Annu. Rev. Mater. Res. 41, 21-40 (2011).

2. Sommerdijk, N. A. J. M. \& Cusack, M. Biomineralization: crystals competing for space. Nat. Mater. 13, 1078-1079 (2014).

3. Reznikov, M., Steele, J. A. M., Fratzl, P. \& Stevens, M. M. A materials science vision of extracellular matrix mineralization. Nat. Rev. Mater. 1, 16041 (2016).

4. Hudson, Z. M. et al. Tailored hierarchical micelle architectures using living crystallization-driven self-assembly in two dimensions. Nat. Chem. 6, 893-898 (2014)

5. Smeets, P. J. M., Cho, K. R., Kempen, R. G. E., Sommerdijk, N. A. J. M. \& De Yoreo, J. J. Calcium carbonate nucleation driven by ion binding in a biomimetic matrix revealed by in situ electron microscopy. Nat. Mater. 14, 394-399 (2015)

6. Wang, Y. et al. Water-mediated structuring of bone apatite. Nat. Mater. 12, 1144-1153 (2013)

7. Nakouzi, E. \& Steinbock, O. Self-organization in precipitation reactions far from the equilibrium. Sci. Adv. 2, e1601144 (2016).

8. Garcia-Ruiz, J. M. et al. Self-assembled silica-carbonate structures and detection of ancient microfossils. Science 302, 1194-1197 (2003).

9. Garcia-Ruiz, J. M., Melero-Garcia, E. \& Hyde, S. T. Morphogenesis of self-assembled nanocrystalline materials of barium carbonate and silica. Science 323, 362-365 (2009).

10. Kellermeier, M., Cölfen, H. \& Garcia-Ruiz, J. M. Silica biomorphs: complex biomimetic hybrid materials from 'sand and chalk'. Eur. J. Inorg. Chem. 32, 5123-5144 (2012)

11. Hyde, S. T., Carnerup, A. M., Larsson, A. K., Christy, A. G. \& Garcia-Ruiz, J. M. Self-assembly of carbonate-silica colloids: between living and non-living form. Phys. A 339, 24-33 (2004).

12. Cölfen, H. \& Antonietti, M. Mesocrystals and non-classical crystallization (John Wiley \& Sons, 2008).

13. Dominguez Bella, S. \& Garcia-Ruiz, J. M. Banding structures in induced morphology crystal aggregates of $\mathrm{CaCO}_{3}$. J. Mater. Sci. 22, 3095-3102 (1987).

14. Nakouzi, E., Ghoussoub, Y. E., Knoll, P. \& Steinbock, O. Biomorph oscillations self-organize micrometer-scale patterns and nanorod alignment waves. J. Phys. Chem. C 119, 15749-15754 (2015).

15. Gal, A., Weiner, S. \& Addadi, L. A perspective on underlying crystal growth mechanisms in biomineralization: solution mediated growth versus nanosphere particle accretion. CrystEngComm 17, 2606-2615 (2015).

16. Wang, G. C., Zhao, X. B., Moller, M. \& Moya, S. E. Interfacial reaction-driven formation of silica carbonate biomorphs with subcellular topographical features and their biological activity. ACS Appl. Mater. Interfaces 7, 23412-23417 (2015).

17. Garcia Ruiz, J. M., Carnerup, A., Christy, A. G., Welham, N. J. \& Hyde, S. T. Morphology: an ambiguous indicator of biogenicity. Astrobiology 2, 353-369 (2002).

18. Bittarello, E., Massaro, F. R. \& Aquilano, D. The epitaxial role of silica groups in promoting the formation of silica/carbonate biomorphs: a first hypothesis. J. Cryst. Growth 312, 402-412 (2010).

19. Kellermeier, M., Melero-Garcia, E., Kunz, W. \& Garcia-Ruiz, J. M. Local autocatalytic co-precipitation phenomena in self-assembled silica-carbonate materials. J. Colloid Interface Sci. 380, 1-7 (2012).

20. Kellermeier, M. et al. Growth behavior and kinetics of self-assembled silica-carbonate biomorphs. Chem. Eur. J. 18, 2272-2282 (2012).

21. Noorduin, W. L., Grinthal, A., Mahadevan, L. \& Aizenberg, J. Rationally designed complex, hierarchical microarchitectures. Science 340, 832-837 (2013).

22. Opel, J. et al. Probing local pH-based precipitation processes in self-assembled silica-carbonate hybrid materials. Nanoscale 7, 17434-17440 (2015).

23. Zhang, X., Rehm, S., Safont-Sempere, M. M. \& Wuerthner, F. Vesicular perylene dye nanocapsules as supramolecular fluorescent $\mathrm{pH}$ sensor systems. Nat. Chem. 1, 623-629 (2009).
24. Zhu, H., Fan, J. L., Du, J. J. \& Peng, X. J. Fluorescent Probes for Sensing and Imaging within Specific Cellular Organelles. Acc. Chem. Res. 49, 2115-2126 (2016).

25. Schulman, S. G., Naik, D. V., Capomacchia, A. C. \& Roy, T. Electronic spectra and electronic structures of some antimicrobials derived from proflavine. J. Pharm. Sci. 64, 982-986 (1975).

26. Nakouzi, E., Knoll, P. \& Steinbock, O. Biomorph growth in single-phase systems: expanding the structure spectrum and $\mathrm{pH}$ range. Chem. Commun. 52, 2107-2110 (2016).

27. Henisch, H. K. Crystals in gels and Liesegang rings (Cambridge University Press, 1998).

28. Zhang, G., Delgado-Lopez, J. M., Choquesillo-Lazarte, D. \& Garcia-Ruiz, J. M. Growth behavior of monohydrocalcite $\left(\mathrm{CaCO}_{3} \cdot \mathrm{H}_{2} \mathrm{O}\right)$ in silica-rich alkaline solution. Cryst. Growth Des. 15, 564-572 (2015).

29. Epstein, I. R. \& Xu, B. Reaction-diffusion processes at the nano- and microscales. Nat. Nanotechnol. 11, 312-319 (2016).

30. Wang, T., Xu, A. W. \& Cölfen, H. Formation of self-organized dynamic structure patterns of barium carbonate crystals in polymer-controlled crystallization. Angew. Chem. Int. Ed. 45, 4451-4455 (2006).

\section{Acknowledgements}

This work was funded by the European Research Council (European Union's Seventh Framework Programme (FP7/2007-2013, grant no. 340863) and the Spanish MINECO grant CGL2010-16882 (co-funded by FEDER). We thank Julian Opel for help with some of the experiments and Isabel Guerra-Tschuschke for technical assistance with electron microscopy.

\section{Author contributions}

M.M., G.Z. and D.G. performed the fluorescence microscopy experiments, analysed the data and discussed the results. J.M. performed the electron microscopy studies and analysed the data. M.K. performed the AFM studies, analysed the data and wrote the paper. M.M. and J.M.G-R. designed the experiments. J.M.G-R. conceived the idea, discussed the results and wrote the paper.

\section{Additional information}

Supplementary Information accompanies this paper at http://www.nature.com/ naturecommunications

Competing financial interests: The authors declare no competing financial interests.

Reprints and permission information is available online at http://npg.nature.com/ reprintsandpermissions/

How to cite this article: Montalti, M. et al. Local $\mathrm{pH}$ oscillations witness autocatalytic self-organization of biomorphic nanostructures. Nat. Commun. 8, 14427 doi: $10.1038 /$ ncomms14427 (2017)

Publisher's note: Springer Nature remains neutral with regard to jurisdictional claims in published maps and institutional affiliations.

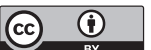

This work is licensed under a Creative Commons Attribution 4.0 International License. The images or other third party material in this article are included in the article's Creative Commons license, unless indicated otherwise in the credit line; if the material is not included under the Creative Commons license, users will need to obtain permission from the license holder to reproduce the material. To view a copy of this license, visit http://creativecommons.org/licenses/by/4.0/

C The Author(s) 2017 